\title{
Time to play, access to attention Addressing one academic library's internal culture around computing tools and skills
}

$\mathbf{L}$ ibrary employees who try developing their skills with computing tools need to practice in order to use their learning in their work. ${ }^{1}$ However, time and space for practice may be elusive. Employees will meet frustration when applying emerging knowledge to problems on the fringes of their learning. Libraries can cultivate employees' budding computing and data skills by sponsoring time to practice, holding space for peers to support each other, and normalizing the struggle inherent in learning and applying digital tools and methods.

This article chronicles efforts at the University of Kansas (KU) Libraries to create space and time for practice, peer support, and judgmentfree attention to employees' efforts to learn and use computing and data tools and skills. ${ }^{2}$ In 2017, KU Libraries' Digital Scholarship Team created and played in the Digital Scholarship Sandbox to explore tools used by researchers to manipulate and analyze data. In 2018, the Sandbox evolved into the Digital Projects Support Hub. The Hub maintained the Sandbox's focus on digital scholarship tools and techniques but modified the group's scope. The Hub continued, with revisions, into 2019 and 2020.

\section{Terms}

In the following descriptions of the Hub, I refer to 2018 as "version one" and 2019 as "version two." Versioning is a concept applied to writing code and text, but seldom to groups. I am emphasizing that this group has tried things, learned, adapted, and recorded its changes from year to year. I use the word regular to describe colleagues who attend most
Hub meetings. The term implies a locus where people gather. The regulars come often, others come occasionally or never.

\section{7: Digital Scholarship Sandbox}

In 2017 KU Libraries employees from the Digital Initiatives and Research and Learning units agreed to meet regularly to explore computing tools related to digital scholarship. We called this experiment the Digital Scholarship Sandbox (Sandbox). The group framed the Sandbox as flexible, ongoing professional development.

The Sandbox held semi-monthly meetings in 2017 and played with data from two internal sources: a 2016 furniture-use study and free text responses from KU Libraries' 2014 LibQUAL+ survey. Our play examined tools and techniques for data visualization, data documentation, text manipulation, and computational text analysis. We drew upon each other's knowledge, software documentation, and lessons from sources such as The Carpentries ${ }^{3}$ and The Programming Historian. ${ }^{4}$

The Sandbox did not complete any defined projects investigating either dataset. This was not our goal. Instead, we wanted to understand what problems different tools could address. The group tackled the task of documenting our changes to the furniture-use data and developed

Jamene Brooks-Kieffer is data services librarian and coordinator of digital scholarship at the University of Kansas Libraries, email: jamenebk@ku.edu

(c) 2021 Jamene Brooks-Kieffer 
empathy for researchers as we concluded that collaborative data documentation is a nightmare. This experience resonates with Jez Cope and James Baker, who underscore expectations that academic library employees will interact with researchers about data and analysis: “. . . learning some of these skills and applying them in our own work is an excellent way to learn the language of researchers and build rapport." ${ }^{\text {" }}$

The open-ended nature of our explorations meant that the group did not articulate research questions for the data we examined. As a result, we struggled to focus our use of a tool deeply enough to derive meaning from that data. We wanted to apply our Sandbox experiences to projects that were driven by one or more research questions. It was time for a change.

The group used the POP (Purpose, Outcome, Process) method for talking through why, what, and how the Sandbox would change. ${ }^{6}$ We wanted to share the Sandbox's lessons with all interested employees: learning new tools is a struggle, incorporating computing into work involves failure, and colleagues can offer support through a frustrating learning curve. These conversations created the Digital Projects Support Hub (Hub).

\section{8: Digital Projects Support Hub, version one}

The Hub emerged with a mission to create and hold a safe time and space for all KU Libraries employees to exchange input about digital projects. We scheduled a prototype meeting in late 2017 to better understand how the Hub would function.

Two colleagues were invited to discuss their projects at the prototype. During the meeting, the group offered resources and advice on challenges the colleagues were experiencing. After the meeting, the Hub asked these colleagues to compare what actually happened in the meeting with what they had thought would happen. Their feedback resulted in two revisions to the Hub's process.

One revision decreased the scope of each meeting to one project. During the prototype, the group's attempts to discuss two different projects prevented fully engaged attention to either. The second revision scoped a project conversation to include talk about goals, stuck places, tool suggestions, and resource recommendations. A project conversation did not obligate anyone present to work on the project outside of a Hub meeting.

After revisions, the Hub held monthly meetings (open to all employees) beginning in 2018. Project conversations addressed internal workflows and research projects, including:

- tracking nominations and awards for Russian literary prizes,

- improving stacks inventory processes,

- automating a manual process for merging data into a web-hosted database,

- linking Twitter data to relevant library resources,

- applying network visualization tools, and

- analyzing text from library chat transcripts.

As the first year drew to a close, the Hub's regulars pondered further evolution of the group's purpose and actions. For 2019, the regulars added a discussion topic option and articulated a goal of attracting more regulars from other units.

\section{9: Digital Projects Support Hub, version two}

In 2019, the Hub offered two possible monthly meeting formats: a project conversation or a discussion topic. Project conversations retained their 2018 format. The new discussion topic format drew from an initial list of ideas from Hub regulars and other employees. The group held an open house in January to encourage participation. Colleagues who had discussed a project with the Hub in 2018 were invited to chat about their experience or submit comments for display during the event.

Version two of the Hub hosted two project conversations. One project involved joining payment and serial holdings data. The other project explored possible research questions about data merged from several internal systems.

Discussion topic meetings were announced in advance to Libraries employees and often in- 
cluded a brief pre-reading. Anecdotal feedback indicated that these discussions were timely and pertinent to employees' work. Topics addressed visualizing, cleaning, and managing data; data privacy; and jargon.

At the end of 2019 and beginning of 2020, Hub regulars again considered possible revisions to the group's purpose, outcomes, and process. As of this writing, revisions for version three include rotating notetaker and facilitator roles among the regulars, and intentional efforts to attract more projects. The Hub continues to gather online during the COVID-19 global pandemic.

\section{Discussion}

\section{Participation}

The Sandbox began with participation from the Libraries' Digital Initiatives and Research and Learning units. Over time, its evolution into the Hub also attracted regular and occasional attendees from Acquisitions and Resource Sharing, Content Development, Cataloging and Archival Processing, the Institute for Digital Research in the Humanities, and International Collections. Through the Hub, people across the organization have an outlet to focus on meeting their need to learn computing and data skills and tools.

\section{Power}

The Sandbox was, and the Hub is, a voluntary group where colleagues explore and discuss. Supervisor, supervisee, staff, pre-tenured, and tenured faculty all attend together. In this setting, the organization's power structures likely echo in our interactions. Over time, the group adopted practices to address this problem; the current version of the Hub retains those who have proven helpful. Meeting agendas are driven by employees' projects or by discussion topics agreed on in advance. Roles such as notetaker and facilitator rotate among the regulars. Meetings and records are open to all Libraries employees.

The Hub applies structured techniques to its discussion topic meetings as one way of coping with the presence of power. The facilitator kicks off the first 30-to- 40 minutes of discussion with a prompt, often from The Discussion Book. ${ }^{7}$ Prompts establish different modes of contributing to a conversation, including writing silently, responding to a question, or reflecting about items on a whiteboard. By offering multiple pathways into a discussion, the Hub attempts to ensure that everyone can express their experiences, skills, and opinions about a topic.

\section{Intentional evolution}

The Sandbox and Hub changed in structure and scope over time as we learned from our experiences in these groups. Our annually scheduled reflections maintain the Hub's overall health. Two example revisions illustrate how the iterative activities we apply to code and writing can benefit organizations.

The Hub's 2017 prototype meeting taught us to set boundaries around what is and is not part of a project conversation. As a result, version one of the Hub articulated that colleagues who brought their ideas and plans to the group must negotiate separately to build a project team. This boundary communicates value and respect for the time and expertise of employees who participate in the Hub.

Version two of the Hub introduced the discussion topic as an alternative meeting format when the group had no project to talk about. This revision kept regulars engaged and attracted new occasional participants. However, discussion topics far outnumbered project conversations in 2019, overshadowing the Hub's founding premise as a resource for our colleagues' projects. The Hub prepared to address this problem in 2020 by contacting individual colleagues and unit leads to seek out and invite more projects, but the COVID-19 pandemic has slowed those plans. In order to further our mission to shift the organization's culture around computing and data skills, the Hub will continue evolving its format and communications online and in person.

\section{Outcomes, or, has the culture changed yet?}

KU Libraries are slowly showing signs that the practices embodied by the Sandbox and the Hub are filtering into the organization's cul- 
ture. Employees from a variety of units participate in informal, exploratory groups, showing that these activities are not tied to one particular person or department.

Late in 2018, a group of employees held an OCR-a-thon. Participants tried open and licensed OCR (Optical Character Recognition) tools on a common set of images, compared the results, and created a publicly accessible guide. ${ }^{8}$ In 2019, I formed a LaTeX study group to learn more about the typesetting system and KU's LaTeX thesis and dissertation template. Python users meet regularly to demonstrate code and access peer-to-peer help. An interest group began meeting in 2018 to explore assessment in the context of a webinar series. ${ }^{9}$ The group remains active and hosts open brown bag discussions about assessment topics and projects.

What would help embed the Hub's practices more deeply into the Libraries' culture? One major change would be for the Libraries' leaders - the dean, members of the leadership team, middle managers- to emphasize that the Hub can be a resource for employees' work. Administrators have quietly encouraged the Hub to continue and evolve, but they could boost the group's impact through a more explicit endorsement.

\section{Conclusion}

If academic libraries expect employees to acquire technology skills in support of campus research, efficient workflows, or some other strategic plan buzz phrase, then the process of learning and support for practicing these skills must be embedded in the organization. Learning and practicing are daily facts in technology work: find a tool, get some data, play around, get stuck, read the documentation, play some more, search the Internet, ask a colleague, try the tool on a problem, rinse, and repeat. But this cycle needs resources: time to explore, community to ask, and access to documentation and adequate system permissions.

Efforts to shift an organization's culture with regard to technology do not have to be personspecific, department-specific, cost money, or involve a consultant. Value the application of technology to library work by reserving space for judgment-free, exploratory peer support. Pay time and attention. Expect revisions. Sponsor this work out loud.

\section{Notes}

1. James Baker, Caitlin Moore, Ernesto Priego, Raquel Alegre, Jez Cope, Ludi Price, Owen Stephens, Daniel van Strien, and Greg Wilson, "Library Carpentry: Software Skills Training for Library Professionals," LIBER Quarterly 26, no. 3 (November 28, 2016): 141-62, https://doi. org/10.18352/lq.10176.

2. The author acknowledges colleagues at KU Libraries without whom the Sandbox and the Hub would not have been possible. Special thanks to Rhonda Houser, Greta Valentine, Rebecca Orozco, Erin Wolfe, and Heather MacBean.

3. The Carpentries, "About The Carpentries Curricula," https://carpentries.org /workshops-curricula/ (accessed October 16, 2019).

4. Programming Historian, "Lesson Directory," https://programminghistorian.org/en /lessons/ (accessed October 16, 2019).

5. Jez Cope and James Baker, "Library Carpentry: Software Skills Training for Library Professionals," International Journal of Digital Curation 12, no. 2 (2017): 267, https://doi.org/10.2218 /ijdc.v12i2.576.

6. Mozilla Foundation, "POP Your Event!" https://foundation.mozilla.org/en/opportunity /open-events-guide/pop-your-event/ (accessed October 16, 2019).

7. Stephen Brookfield and Stephen Preskill, The Discussion Book: 50 Great Ways to Get People Talking (San Francisco, CA: Jossey-Bass, 2016).

8. University of Kansas Libraries, "Subject \& Course Guides: Optical Character Recognition (OCR)—Getting Started," https://guides. lib.ku.edu/ocr (accessed January 29, 2020).

9. WebJunction and OCLC Research, "Webinar Series: Evaluating and Sharing Your Library's Impact," October 18, 2018, https://www. webjunction.org/news/webjunction/webinar -series-research-assessment.html (accessed January 29,2020$)$. 